\title{
Magnetic Resonance Imaging in Assessment of Recurrent Rectal Malignancy in Iraqi Patients
}

\author{
Dr. Khaleel Ibraheem Mohson ${ }^{1}$, Anas K. Awn ${ }^{2}$ \\ ${ }^{1} \mathrm{MBChB}$, DMRD, CABMS (RAD), Lecturer, Iraqi National Cancer Research Center, University of Baghdad \\ ${ }^{2} \mathrm{MBChB}$, Iraqi National Cancer Research Center, University of Baghdad
}

\begin{abstract}
Background: The incidence of colorectal cancer has been increasing dramatically following thefinancial and economic advancements. Now, colorectal cancer is the third foremost cause of cancer deaths in both males and femalesin the United States.Many diagnostic tools had been emerging in the screening and early diagnosis of the disease including fecal occult blood and colonoscopy, but from a radiological viewpoint; Magnetic Resonance (MR) imaging is still by far the primary cross-sectional imaging modality used for preoperative local staging of rectal cancer, patient-follow up after rectal surgery and neoadjuvant chemotherapy due to its high spatial resolution and marked soft-tissue characterization. Objective: The objective of this studyis to assess the role of MR imaging in recurrent rectal tumor detection, its site, signal characteristics and appearance on variable sequences and whether or not the tumor is resectable. Patients and method: This is a retrospective study compromised of 50 patients; it was performed during the period of November 2016 to September 2017 in MR unit in the Oncology Teaching Hospital, Medical City Compound, where the patients were referred from the Oncology Clinic then their imaging findings were assessed by specialist radiologists. Results: A review of the study that compromised of 50 patients with rectal cancer and underwent surgery; 20 were males and 30 were females, their age ranges between $28-85$ years, 12 patients (24\%) showed no recurrence while the remaining 38 patients (76\%) showed recurrence. However, ofthe recurrent tumor patients; 12 had recurrence at ileoanal anastomosis site in which 5 had intramural (41\%), 5 hadextraluminal (41\%) and 2 patients had both intra- and extramural recurrence. While in patients with abdominoperineal resection; 9 had anterior local recurrence (34.6\%) and 7 cases of presacral regrowth were found (27\%), only 3 patients showed lateral pelvic wall muscular invasion (11.5\%). Also, lymph nodes recurrence was found in 7 patients mainly involving the mesorectal group (57\%). The obturator group involved in (28\%) and least infiltrated nodes were the inguinal group in (15\%). The results of the MR signal in corresponding to nearby pelvic muscles on T1 and T2 weighted images were isointense in $68 \%$ and greater than in $32 \%$, while the enhancement pattern after intravenous Gadolinium is equal to ileum wall enhancement in $76 \%$ and greater than in $24 \%$. Conclusion: MR imaging is thepreferred imaging modality inevaluation and patient- follow up with suspicious recurrence after rectal malignancy surgery.Its value was demonstrated in characterization and diagnosis of pelvis lesion(s) by assessing their appearance on T1,T2 and fat suppression T2 MR sequences, as well as assessing their enhancement pattern after intravenous Gadolinium in fat suppression sequence.
\end{abstract}

Keywords: rectal tumor, recurrence pattern, MR imaging, Gadolinium fat suppression T1 ,pelvic muscles signal.

\section{Introduction}

MR imaging is the leading imaging modality employed for preoperative and postoperative staging of primary rectal cancer and assessing it recurrence and thus its ability to differentiate the completely curable and stable from progressive cancer in the follow up of the primary rectal cancer status after chemotherapy and radiotherapy [1,2].

Local recurrence of rectal cancer after resection is associated with high patient morbidity and mortality[3]. The expected percentage of local recurrence varying from $3 \%$ to $32 \%$ in different studies [4,5]. Also, factors that control the degree of local recurrence include tumor stage, grade and the rightadministration ofappropriate treatment $[6,7,8]$.

Most local recurrences of rectal cancer happen within the first 3 years of primary surgery [5]. Early detection of local recurrence is vital in preventing progression to a state beyond respectability $[9,10]$. Since recurrence is asymptomatic in $23 \%-30 \%$ of cases; regular follow-up imaging can facilitate early detection [11].

Recurrences may be classified as either central, anastomotic recurrence after resection, local recurrence at the primary site after transanal excision, and perineal recurrence; (b) anterior, in this case; the recurrence invades the bladder, uterus, prostate, vagina, or seminal vesicles; (c) posterior, in this case, it involves the invasion of the sacrum and coccyx or involvement of the presacral fascia; or (d) lateral, the tumor in this region penetrates the bony pelvic sidewall or the iliac vasculature, terminal ureters, lateral lymph nodes, nerves in pelvic floorand musculature [12].

The role of imaging is dual; firstly, the radiologicevaluation is used to determine whether the recurrent disease is restricted to the pelvis or extended outside. Secondly, the imaging is used to determine the local extent of the recurrent disease and its localization within the pelvis to help surgeons determine the probability of resection and plan the best approach [12]. The tumor recurrence and the postoperative fibrosis remain a dilemma for MR in its ability to reach the final diagnosis and accurate differentiation because the fibrosis usually shows angular margin and in contrast to recurrence that often has a rounded margin as in the condition for post radiation changes and probable invasion of nearby structure still major issue.However, the interval of the increase in tumor size over time at serial follow-up imaging is helpful in differentiating postoperative and postradiation therapy changes from tumor recurrence [13].

\section{Patients and method}

This is a retrospective study compromised of 50 patients; it was performed during the period of November 2016 to September 2017 in MR unit in the Oncology Teaching

\section{Volume 6 Issue 12, December 2017}




\section{International Journal of Science and Research (IJSR) \\ ISSN (Online): 2319-7064}

Index Copernicus Value (2016): 79.57 | Impact Factor (2015): 6.391

Hospital, Medical City Compound, where the patients were referred from the Oncology Clinic then their imaging findings were assessed by specialist radiologists. The study sample includes twenty malesand thirtyfemales, whom age range from $28-85$ years.

Those patients were diagnosed to have rectal adenocarcinoma by colonoscopy and were operated upon by resection or anal anastomosis or had abdominoperineal resection and after that they were referred to oncologists for their full assessment and treatment. The oncologists on other hand sent them to the radiological unit including ultrasound and MR. Furthermore, in the MR unit the patients underwent pelvis MRI using Siemens 1.5 Tesla machine, the patients were examined in supine position the coil is wrapped around their pelvis and the examination was done from the iliac crest to perineum using $\mathrm{T} 1, \mathrm{~T} 2, \mathrm{~T} 2$ fat suppression sequences, the sequences were obtained in axial and coronal plain, then intravenous contrast was injected using Gadolinium and the images acquired in fat suppression sequence. TheGadolinium was used in a dose of $0.1 \mathrm{mmol} / \mathrm{kg}$ body weight, patients with renal failure were not given the contrast. The presence or absence of local pelvic lesion (s), their signal intensity in different sequences and the pattern of enhancement were assessed, the presence or absence of adenopathy (depending on the use of lymph node short-axis diameter of $10 \mathrm{~mm}$ or greater and shape of it to identify suspicious lymph nodes) and their regions and local pelvis organs invasion were also evaluated, then the patients with MR diagnosed to have local recurrence were subjected to follow up MR scan to assess the response to treatment whether chemo- or radiotherapy.

\section{Results}

Our study is a review study composed of 50 patients, $40 \%$ are males and $60 \%$ are females. The age ranges between 28 85 years with the mean 51.1 years, the sex and age distribution is discussed in (figure 1 and table 1)

Table 1: Age distribution

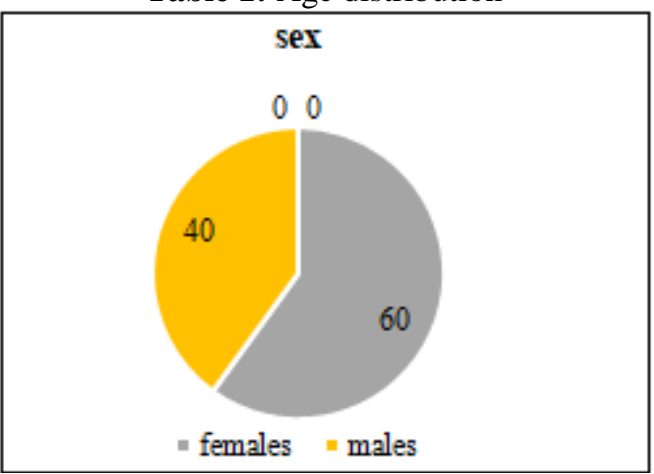

Figure 1: Sex distribution

According to contrast enhanced MR imaging with detailed examination; no locoregional recurrence was seen in 12 patients while clear recurrence was noticed in 38 patients as shown in figure 2 .

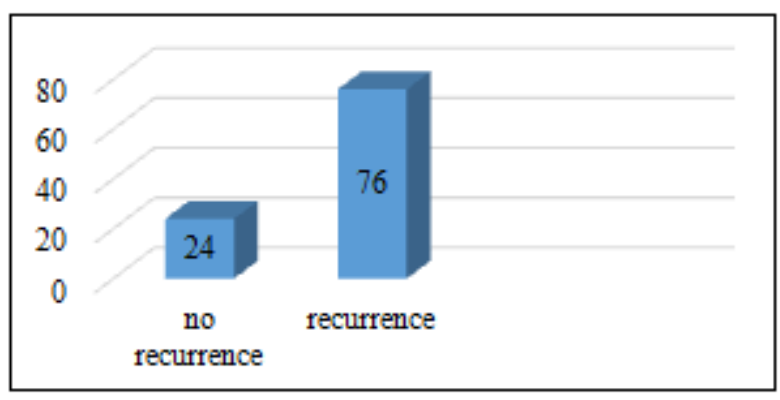

Figure 2: recurrence versus no recurrence by contrast enhanced MR imaging

Regarding local recurrence, it was divided according to the type of surgery, the recurrence following the tumor resection. End to end anastomosis was seen in 12 patients (31\%)of them. While, $41 \%$ had intramural recurrence, $41 \%$ extramural regrowth, combined recurrence was seen in $18 \%$, table 2 .

Table 2: Recurrence after tumor resection and end to end anastomosis:

\begin{tabular}{|l|l|}
\hline Recurrence site & No. \\
\hline Intramural & $5(41 \%)$ \\
\hline Extramural & $5(41 \%)$ \\
\hline Combined & $2(18 \%)$ \\
\hline Total & 12 \\
\hline
\end{tabular}

Of those who had abdominoperineal resection, recurrence was observed in 26 of themdistributed in the following sites; anterior in 9 patients $(34.6 \%)$, presacral in 7 patients $(27 \%)$, Lymph nodes deposit was seen in 7 patients $(27 \%)$ and finally lateral recurrence within the pelvic lateral muscles was seen in 3 patients which represents $(11.5 \%)$, detailed list of site of recurrence is shown in table 3 below.

Table 3: Local recurrence sites after abdominoperineal resection of rectal tumor:

\begin{tabular}{|l|lc|}
\hline Recurrence site & No. & \\
\hline Anterior & Cervix and vagina 7/9 (78\%) \\
& Prostate & $2 / 9(22 \%)$ \\
\hline Presacral & $7 / 26(27 \%)$ & \\
\hline Lymph nodes & Mesorectal & $4 / 7(57 \%)$ \\
& Obturator & $2 / 7(28 \%)$ \\
& Inguinal & $1 / 7(15 \%)$ \\
\hline Lateral & & $3 / 26(11.5 \%)$ \\
\hline
\end{tabular}

Regarding the MR imaging findings of recurrent rectal tumor, the recurrence is isointense in the skeletal pelvic muscle on $\mathrm{T} 1$ and $\mathrm{T} 2$ weighted images in 26 patients out of 38 patients $(68 \%)$, while being lower than muscles on $\mathrm{T} 2$ in remaining 12 patients $(32 \%)$.

After Intravenous injection of Gadolinium; most of the recurrent tumors depict enhancement similar to small bowel pelvic ileal loops which represents $76 \%$. On top of that; the remaining $24 \%$ had enhancement greater than that of bowel. Detail finding of MR imaging finding and enhancement patterns are shown in table 4 and figure 3 respectively. 


\section{International Journal of Science and Research (IJSR) \\ ISSN (Online): 2319-7064}

Index Copernicus Value (2016): 79.57 | Impact Factor (2015): 6.391

Table 4: signal characteristic of recurrent rectal tumor on MR images:

\begin{tabular}{|c|c|}
\hline Signal of recurrent tumor & No. \\
\hline Equal to muscle signal & $26(68 \%)$ \\
\hline Greater than muscle signal & $12(32 \%)$ \\
\hline Total & $38(100 \%)$ \\
\hline
\end{tabular}

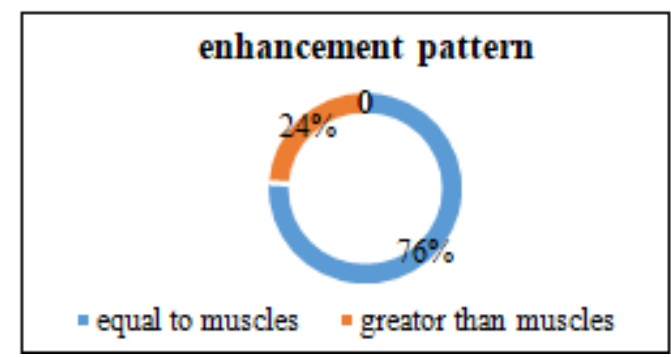

Figure 3: pattern of recurrent tumor enhancement.

\section{Discussion}

Colorectal cancer, by far, is the most frequent type of gastrointestinal tract malignancyand thus being a leading cause of illness and death in developed nations [Sahani D V, Kalva S P, Hahn P F. Imaging of rectal cancer. Semin Radiat Oncol. 2003; 13(4):389-402.]. Rectal adenocarcinoma accounts for 40 to $50 \%$ of colorectal cancers, with a substantialdanger of locoregional recurrence and distant metastases. [Beets-Tan R G, Beets G L. Rectal cancer: review with emphasis on MR imaging. Radiology. 2004; 232(2):335-346.]Multiple treatment possibilities areavailable for rectal cancer, depending on the tumor stage and the patient'sgeneral health condition. Complete resection is curative with the surgical approach being dependent on tumor location and extent[ Iafrate F, Laghi A, Paolantonio P, et al. Preoperative staging of rectal cancer with MR imaging: correlation with surgical and histopathologic findings. Radiographics. 2006; 26(3):701-714.].

In thisstudy no local recurrence was observed in 12 patients in whom we reviewed the histopathological and MR imaging reports which revealed that; the tumor was limited inner to lamina properia in 6 of them, while 4 of them had only polypoidal mucosal lesion, while in the last 2; the tumor caused a bulge of lamina properia with minimal fatty stranding in mesorectal fatty tissue.

Nonetheless, the tumor in the remaining 38 patients was observed to be locally recurrent at the site of anastomosis and it waseither seen as intramural growth in $41 \%$, extramural in $41 \%$ or combined in $18 \%$. Also, it appeared as nearly well-defined heterogeneous enhancing lesion in 9 of them, the remaining 3 showed tumor with necrotic center and variable peripheral mural thickening and enhancing, those were referred to a surgeon.

In the 26 patients with abdominoperineal resection; 9 patients had anterior recurrence, while the uterine cervix and vagina were involved in 7 patients, while the bladder and prostate were involved in only 2 patients. In addition to that, 7 patients had presacral recurrence who revealed thickening and heterogeneously enhanced presacral fascia up to $2^{\text {nd }}$ sacral vertebra, follow-up MR two months after chemotherapy revealed reduction in tumor size, lymph nodes invasion was seen as rounded lost hilum heterogeneously enhanced lymph nodes involving the mesorectal group in 4/7 patients,obturator group in $2 / 7$ patients and one patient showed inguinal lymphadenopathy in whom fine needle aspirate revealed adenocarcinoma cells.

The pattern of enhancement of the recurrent tumor is either similar to bowel wall in the majority of patients or greater than in the remaining ones and that is in accord withpema PJ etal [14], the T2 weighted image signal is similar to muscles in most of patients while greater in a few of them and that is in accord with Dresen RC etal [15].

\section{Conclusion}

MR imaging is a vitalimaging technique for theearly detection of locoregional recurrence of rectal adenocarcinoma,MR imaging similarly offers a preoperative scheme for surgeons to decide whether the tumor is resectable or not after primary surgery.

\section{References}

[1] Kim DJ, Kim JH, Lim JS, et al. Restaging of rectal cancer with MR imaging after concurrent chemotherapy and radiation therapy. RadioGraphics 2010; 30(2):503516.

[2] Barbaro B, Vitale R, Leccisotti L, et al. Restaging locally advanced rectal cancer with MR imaging after chemoradiation therapy. RadioGraphics 2010; 30(3):699-716.

[3] Tudorica A, Thomas CR Jr, Huang W. Invited commentary. RadioGraphics2010;30(3):716-719; discussion 719-721.

[4] Beets-Tan RG, Beets GL. Local staging of rectal cancer: a review of imaging. J MagnReson Imaging 2011;33(5):1012-1019.

[5] Nesbakken A, Nygaard K, Westerheim O, Mala T, Lunde OC. Local recurrence after mesorectal excision for rectal cancer. Eur J SurgOncol 2002;28 (2):126-134.

[6] Kapiteijn E, Putter H, van de Velde CJ; Cooperative investigators of the Dutch ColoRectal Cancer Group. Impact of the introduction and training of total mesorectal excision on recurrence and survival in rectal cancer in the Netherlands. Br J Surg 2002;89(9):11421149.

[7] Nelson H, Sargent DJ. Refining multimodal therapy for rectal cancer. N Engl J Med 2001;345(9): 690-692.

[8] Heriot A, Kumar D. Rectal cancer recurrence: factors and mechanisms. Colorectal Dis 2000;2(3): 126-136.

[9] Palmer G, Martling A, Cedermark B, Holm T. A population-based study on the management and outcome in patients with locally recurrent rectal cancer. Ann SurgOncol 2007;14(2):447-454.

[10] Bouchard P, Efron J. Management of recurrent rectal cancer. Ann SurgOncol 2010;17(5):1343-1356.

[11] Heriot AG, Byrne CM, Lee P, et al. Extended radical resection: the choice for locally recurrent rectal cancer. Dis Colon Rectum 2008;51(3):284-291.

[12] Moore HG, Shoup M, Riedel E, et al. Colorectal cancer pelvic recurrences: determinants of resectability. Dis Colon Rectum 2004;47(10):1599-1606.

\section{Volume 6 Issue 12, December 2017}




\section{International Journal of Science and Research (IJSR) \\ ISSN (Online): 2319-7064}

Index Copernicus Value (2016): 79.57 | Impact Factor (2015): 6.391

[13] Tan PL, Chan CL, Moore NR. Radiological appearances in the pelvis following rectal cancer surgery. ClinRadiol 2005;60(8):846-855.

[14] Pema PJ, Bennett WF, Bova JG, Warman P. CT vs MRI in diagnosis of recurrent rectosigmoid carcinoma. J Comput Assist Tomogr 1994;18(2):256-261

[15] Dresen RC, Kusters M, Daniels-Gooszen AW, et al. Absence of tumor invasion into pelvic structures in locally recurrent rectal cancer: prediction with preoperative MR imaging. Radiology 2010;256(1): 143-150.

Volume 6 Issue 12, December 2017

www.ijsr.net 